\title{
Geometric nature of the environment-induced Berry phase and geometric dephasing
}

\author{
Robert S. Whitney, ${ }^{1}$ Yuriy Makhlin, ${ }^{2,3}$ Alexander Shnirman, ${ }^{4}$ and Yuval Gefen ${ }^{5}$ \\ 1 Departement de Physique Théorique, Université de Genève, \\ 24 quai Ernest-Ansermet, 1211 Genève 4, Switzerland. \\ ${ }^{2}$ Institut für Theoretische Festkörperphysik, Universität Karlsruhe, D-76128 Karlsruhe, Germany \\ ${ }^{3}$ Landau Institute for Theoretical Physics, Kosygin st. 2, 117940 Moscow, Russia. \\ ${ }^{4}$ Institut für Theoretische Festkörperphysik, Universität Karlsruhe, D-76128 Karlsruhe, Germany. \\ ${ }^{5}$ Department of Condensed Matter Physics, The Weizmann Institute of Science, Rehovot 76100, Israel.
}

(Dated: January 26, 2005)

\begin{abstract}
We investigate the geometric phase or Berry phase (BP) acquired by a spin-half which is both subject to a slowly varying magnetic field and weakly-coupled to a dissipative environment (either quantum or classical). We study how this phase is modified by the environment and find that the modification is of a geometric nature. While the original BP (for an isolated system) is the flux of a monopole-field through the loop traversed by the magnetic field, the environment-induced modification of the BP is the flux of a quadrupole-like field. We find that the environment-induced phase is complex, and its imaginary part is a geometric contribution to dephasing. Its sign depends on the direction of the loop. Unlike the BP, this geometric dephasing is gauge invariant for open paths of the magnetic field.
\end{abstract}

PACS numbers: 03.65.Vf, 03.65.Yz, 85.25.Cp

Introduction. The Berry phase (BP) is a fundamental quantum-mechanical phenomenon related to the adiabatic theorem. Berry [1] showed that the phase acquired by an eigenstate of a slowly varying Hamiltonian $H(t)$ is related to the geometric properties of the loop traversed by $H(t)$. In the presence of dissipation the condition of adiabaticity and the existence of the Berry phase require careful analysis. The widespread criterion of adiabaticity, based on a comparison of the rate of change of the Hamiltonian with the gap in the spectrum should be modified to involve the matrix elements of the system-environment coupling, cf. 2]. Here we study the interplay of the varying field and the dissipation, analyzing the BP in the limiting case of weak system-environment coupling. This analysis is relevant to the recent and proposed experiments to manipulate quantum two-level systems (qubits). Our findings could be tested in solid-state qubits, such as superconducting nanocircuits [3, 4, [5, 6].

Berry [1] considers a two-level spin-half system in a magnetic field [7], which is varied slowly along a closed path: $H_{\text {spin }}=-\frac{1}{2} \mathbf{B}(t) \boldsymbol{\sigma}$. The rate of the field's change is characterized by the time to complete the loop, $t_{\mathrm{P}}$. In the adiabatic limit, $B t_{\mathrm{P}} \gg 1$, the relative phase acquired by the eigenstates is a sum $\Phi=\oint|\mathbf{B}(t)| d t+\Phi_{\mathrm{BP}}$ of the dynamical and Berry phases. The latter is geometric, it depends on the geometry of the loop but not on the details of its traversal (for an isolated spin-half it is given by the solid angle subtended by the loop $\mathbf{B}(t)$ ). In the spin language, the evolution is a rotation of the spin by an angle $\Phi$ about $\mathbf{B}$.

If the spin is not isolated, the dynamics are more complicated. For a static field, B, dissipation induces energy and phase relaxation processes (with the time-scales $T_{1}, T_{2}$ respectively) and a Lamb-like shift of the level splitting, $\delta B_{\text {Lamb }}$, which modifies the dynamical phase. (a) field $\delta \mathbf{a}(\mathbf{B})$ (solid arrows)

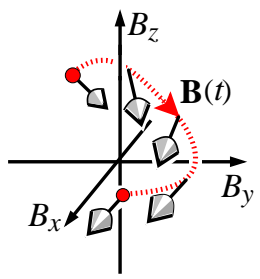

(b) quadrupole-like field $\delta \mathbf{b}(\mathbf{B})=\nabla \times \delta \mathbf{a}(\mathbf{B})$

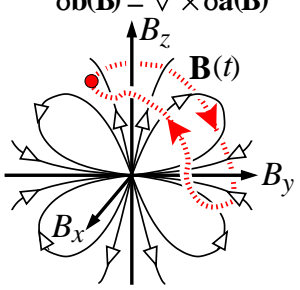

FIG. 1: (a) The field $\delta \mathbf{a}(B)$, see eq. (13), in the vicinity of an open path of $\mathbf{B}(t)$ (the dotted line). The environmentinduced modification of the Berry phase, $\delta \Phi_{\mathrm{BP}}$, is the integral of $\delta \mathbf{a}(B)$ along this path. For such open-paths, the geometric dephasing is gauge-invariant (unlike the phase). (b) The quadrupole-like field $\delta \mathbf{b}(B)$, see eqs. (14 15), $(\delta \mathbf{b}(B)$ is cylindrically symmetric about the $B_{z}$-axis). For a closed path of $\mathbf{B}(t), \delta \Phi_{\mathrm{BP}}$ is given by the flux of $\delta \mathbf{b}(B)$ through the path.

Strong dephasing masks the BP, however it can be observed if $B \gg T_{2}^{-1}$ [2]. In this weak-coupling limit one can carry out a BP experiment slowly enough for nonadiabatic effects to be ignored $\left(t_{\mathrm{P}} \gg B^{-1}\right)$ while ensuring it is fast enough that dephasing has not destroyed all phase information $\left(t_{\mathrm{P}} \lesssim T_{2}\right)$.

In this letter we show that the coupling to the environment modifies the $\mathrm{BP}$ when the magnetic field is slowly varied. In the adiabatic limit this modification, $\delta \Phi_{\mathrm{BP}}$, is geometric (see Fig. [1] and complex [8]. Its real part is an environment-induced $\mathrm{BP}$, thus the total $\mathrm{BP}$ is $\Phi_{\mathrm{BP}}^{(0)}+\operatorname{Re}\left[\delta \Phi_{\mathrm{BP}}\right]$ (where $\Phi_{\mathrm{BP}}^{(0)}$ is the $\mathrm{BP}$ for an isolated spin-half). Its imaginary part, $\operatorname{Im}\left[\delta \Phi_{\mathrm{BP}}\right]$, is a geometric correction to dephasing, whose sign depends on the direction of the loop. The magnitude of coherency will thus depend on the sign of the loop's winding number. Notably $\operatorname{Im}\left[\delta \Phi_{\mathrm{BP}}\right]$ is gauge-invariant for open as well as 
closed paths of the $B$-field.

Specifically we study the environment-induced BP for an arbitrary path $\mathbf{B}(t)$, allowing us to analyze its geometric nature. For an isolated spin-half the Berry phase is given by the flux through the closed loop $\mathbf{B}(t)$ of the field $\mathbf{b}_{\mathfrak{m}}(\mathbf{B})$ of a charge-one monopole at the origin in $\mathbf{B}$ space 1]: we show that the environment-induced modification may be interpreted similarly and find the corresponding field distribution $\delta \mathbf{b}(\mathbf{B})$ in the $\mathbf{B}$-space. This field (and thus $\delta \Phi_{\mathrm{BP}}$ ) scales quadratically with the spinenvironment coupling, similarly to the dissipative rates, $T_{1}^{-1}, T_{2}^{-1}$. If the environment-induced field fluctuates along a single direction, $z$, the field $\delta \mathbf{b}(\mathbf{B})$ is axially symmetric, has no axial component, $\delta b_{\varphi}=0$, and the angular distribution of a quadrupole. The field $\delta \mathbf{b}(\mathbf{B})$ is given by a sum over frequencies of the environment modes (for the lowest non-trivial order in the strength of the spinenvironment coupling). The contribution of low frequencies $\Omega \ll B$ to $\delta \mathbf{b}$ is exactly the field of a quadrupole, while high-frequency modes have the angular dependence of a quadrupole but a different radial dependence.

Let us emphasize the novel points of our analysis in comparison to earlier work on BP in systems coupled to quantum or classical environments [2, 9, 10, 11]. Some of these works have focused on the visibility of BP in spite of the dephasing, they did not find the modification of the BP. In [9] it is absent because the Master equation used there neglects the effect of $\dot{\mathbf{B}} \neq 0$ on the dissipative rates. A modification of the $\mathrm{BP}$ was found in [2], however the time-dependence of $\mathbf{B}(t)$ used there was too specific to see the geometric nature of this modification. Here we are able to consider a totally arbitrary (slowly varying) $B(t)$ and show, for the first time, that the modification to the $\mathrm{BP}$ is geometric. We also observe that the effect of the $\mathrm{BP}$ on the dephasing rate is geometric.

To find the BP we analyze the (directly observable) phase factor in the evolution operator for a given field dynamics $\mathbf{B}(t)$. In this phase we attribute to $\mathrm{BP}$ the contributions independent of $t_{\mathrm{P}}$ [12] (those $\propto t_{\mathrm{P}}$ are part of the dynamical phase, and the terms $\mathcal{O}\left(1 / t_{\mathrm{P}}\right)$ are nonadiabatic corrections [13|).

For open paths the BP is gauge-dependent 14, we show that this is not the case for the geometric dephasing, $\operatorname{Im}\left[\delta \Phi_{\mathrm{BP}}\right]$. The gauge-dependence of the $\mathrm{BP}$ is a consequence of ambiguity in the choice of instantaneous basis for a given $\mathbf{B}(t) ; \mathbf{B}(t)$ defines the instantaneous $\tilde{z}$-axis but the $\tilde{x}$-axis may lie anywhere in the plane perpendicular to $\mathbf{B}(t)$. Gauge transformations rotate the $\tilde{x}$-axis in this plane. The ambiguity is absent for closed loops, where the final basis must coincide with the initial one. In addition one can monitor the magnitude of the transverse spin component (dephasing); this magnitude is independent of the choice of $\tilde{x}$-axis, i.e. the dephasing (and corrections to it) is gauge-invariant even for open paths.

Berry phase for an isolated spin. We evaluate the

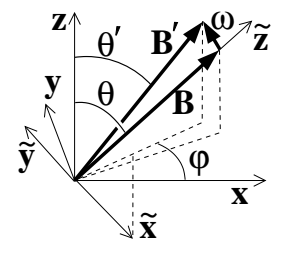

FIG. 2: The laboratory $(x, y, z)$ and rotating $(\tilde{x}, \tilde{y}, \tilde{z})$ frames.

BP for an isolated spin-half [1] using a rotating frame (RF) 15], the one we choose (see Fig. 21) has its $\tilde{z}$-axis along the field $\mathbf{B}(t)$ and $\boldsymbol{e}_{\tilde{y}} \perp \boldsymbol{e}_{z}$ (then $\boldsymbol{e}_{\tilde{x}}\left(t_{\mathrm{P}}\right)=\boldsymbol{e}_{\tilde{x}}(0)$ ). In this frame the magnetic field is $\mathbf{B}^{\prime}=\mathbf{B}+\boldsymbol{\omega}$, where the angular velocity of the RF is

$$
\boldsymbol{\omega}=\dot{\theta} \boldsymbol{e}_{\tilde{y}}+\dot{\varphi} \boldsymbol{e}_{z}
$$

Transforming to the RF gives the Hamiltonian, $\tilde{H}=$ $U H_{\text {spin }} U^{-1}+i \dot{U} U^{-1}=-\frac{1}{2}(\tilde{\mathbf{B}}+\tilde{\boldsymbol{\omega}}) \hat{\boldsymbol{\sigma}}$, where the transformation $U=\exp \left(i \theta \hat{\sigma}_{y} / 2\right) \exp \left(i \varphi \hat{\sigma}_{z} / 2\right)$ (with $B, \theta, \varphi$ being spherical coordinates of $\mathbf{B}(t))[16] ; \tilde{\mathbf{B}}=(0,0, B)$ and $\tilde{\boldsymbol{\omega}}=\left(\omega_{\tilde{x}}, \omega_{\tilde{y}}, \omega_{\tilde{z}}\right)=(-\sin \theta \dot{\varphi}, \dot{\theta}, \cos \theta \dot{\varphi})$ are coordinates in the RF. The evolution in the RF is a rotation of the spin about the $\tilde{z}$-axis by the angle $\oint d t\left|\mathbf{B}^{\prime}(t)\right|$. To lowest order in $\omega$ the accumulated phase is $\int d t\left(B(t)+\omega_{\tilde{z}}\right)$ 17]. Subtracting the dynamical phase, we find the BP;

$$
\Phi_{\mathrm{BP}}=\int d t \omega_{\tilde{z}}(t)=\oint d \varphi \cos \theta=\oint d \mathbf{B} \mathbf{a}(\mathbf{B}) .
$$

In the chosen frame (gauge) the 'vector potential' a has only one non-zero spherical component $a_{\varphi}=1 /(B \tan \theta)$. Stokes' theorem is used to writing eq (2) as

$$
\Phi_{\mathrm{BP}}=\int d \mathbf{S} \mathbf{b}(\mathbf{B}), \quad \mathbf{b}(\mathbf{B}) \equiv \nabla_{\mathbf{B}} \times \mathbf{a}(\mathbf{B}),
$$

where $\nabla_{\mathbf{B}}$ denotes derivatives w.r.t. the components of $\mathbf{B}$, and the field $\mathbf{b}(\mathbf{B})$ is that of a charge-one monopole, $b_{B}=1 / B^{2}$.

Environmental contribution to $B P$. Adding a noisy environment-induced field $\hat{\mathbf{X}}(t) \equiv \hat{X}(t) \boldsymbol{e}_{z}$, the Hamiltonian reads (in the lab frame) []

$$
\hat{H}=-\frac{1}{2} \mathbf{B}(t) \hat{\boldsymbol{\sigma}}-\frac{1}{2} \hat{X} \hat{\sigma}_{z}+\hat{H}_{\mathrm{env}}(\hat{X}) .
$$

This Hamiltonian models a situation in which the spinenvironment coupling is strongly anisotropic. This is often true for solid-state qubits, where various 'spin' components couple to entirely different environmental degrees of freedom [3, 4, 5]. Our analysis is easily generalized for multi-directional coupling [18]. Below we express our results in terms of the statistical properties (correlators) of the environment's noise, $\hat{X}(t)$. We consider any environment which gives rise to Markovian evolution, i.e. $\langle\hat{X}(t) \hat{X}(0)\rangle$ decays on a timescale $\tau \ll t_{\mathrm{P}}, T_{\text {diss }} \equiv \min \left(T_{1}, T_{2}, \delta B_{\text {Lamb }}^{-1}\right)$ however we do not assume $\tau \ll B^{-1}$. We further consider weak dissipation 
such that $B T_{\text {diss }} \gg 1$. In the $\mathrm{RF}$ the Hamiltonian reads: $\tilde{H}=-\frac{1}{2} B(t) \hat{\sigma}_{z}-\frac{1}{2} \tilde{\boldsymbol{\omega}}(t) \hat{\boldsymbol{\sigma}}-\frac{1}{2} \hat{X}\left(\cos \theta \hat{\sigma}_{z}-\sin \theta \hat{\sigma}_{x}\right)+\hat{H}_{\mathrm{env}}$.

We analyze this system in the eigen-basis of $B(t) \hat{\sigma}_{z}+$ $\tilde{\boldsymbol{\omega}}(t) \hat{\boldsymbol{\sigma}}$, with the total field $B^{\prime}(t) \approx B(t)+\omega_{\tilde{z}}$ and the new angle $\theta^{\prime}$ as defined in Fig. 2

The small terms $\propto \omega,\left\langle\hat{X}^{2}\right\rangle$ induce corrections to the evolution. As discussed above, the correction $\propto \omega$ to the rate of phase accumulation yields the $\mathrm{BP}$, while the correction $\propto\left\langle\hat{X}^{2}\right\rangle$ gives $T_{1}^{-1}, T_{2}^{-1}, \delta B_{\text {Lamb }}$. Here we evaluate the environment-induced $\mathrm{BP} \propto\left\langle\hat{X}^{2}\right\rangle \omega$. To this end, we reduced the problem with a time-dependent field to a problem with a stationary field (to leading order [17]) by going to the RF and then use the standard formalism for evaluation of dissipative effects.

Consider the kinetic equation for the reduced density matrix of the spin $\rho$. Iteration of the Liouville equation 19, 20, 21, 22, 23] leads to a Dyson-type master equation:

$$
\dot{\rho}_{i j}(t)=\frac{\mathrm{i}}{2}\left[B^{\prime} \sigma_{z}, \rho(t)\right]_{i j}+\int_{0}^{t} d t_{1} \Sigma_{i j, i^{\prime} j^{\prime}}\left(t, t_{1}\right) \rho_{i^{\prime} j^{\prime}}\left(t_{1}\right) .
$$

The 'self-energy' $\Sigma\left(t, t_{1}\right)$ can be evaluated perturbatively in $\hat{X}$; diagrammatically it is the sum of irreducible diagrams [22, 23]. For short-correlated noise $\tau \ll T_{\text {diss }}$, one can make a Bloch-Redfield approximation 20, 21] giving Markovian evolution. We need only consider the off-diagonal density matrix element, $\rho_{12}(t)$, as it contains all phases (and dephasing) . After a secular approximation (for $\Gamma \ll B^{\prime}$ ) 24], it is given by [25]

$$
\begin{gathered}
\dot{\rho}_{12}(t)=\left[\mathrm{i} B^{\prime}(t)+\Gamma(t)\right] \rho_{12}(t), \\
\Gamma(t) \equiv \int_{-\infty}^{t} d t_{1} \Sigma_{12,12}\left(t, t_{1}\right) \exp \left(-\mathrm{i} \int_{t_{1}}^{t} B^{\prime}(\tau) d \tau\right)
\end{gathered}
$$

We follow 20, 21, 22, 23] evaluating $\Sigma_{12,12}$ to lowest order $\left(2^{\text {nd }}\right.$-order $)$ in $\hat{X}$. This "golden-rule" calculation yields

$$
\begin{aligned}
\Gamma(t)= & -\int_{-\infty}^{t} d t_{1} S\left(t-t_{1}\right)\left[\cos \theta^{\prime}(t) \cos \theta^{\prime}\left(t_{1}\right)\right. \\
& \left.+\frac{1}{2} \sin \theta^{\prime}(t) \sin \theta^{\prime}\left(t_{1}\right) \exp \left(-\mathrm{i} \int_{t_{1}}^{t} B^{\prime}(\tau) d \tau\right)\right],
\end{aligned}
$$

where $S\left(t-t_{1}\right) \equiv(1 / 2)\left\{\left\langle\hat{X}(t) \hat{X}\left(t_{1}\right)\right\rangle+\left\langle\hat{X}\left(t_{1}\right) \hat{X}(t)\right\rangle\right\}$ is the symmetrized environment correlator 26]. If we neglect all order- $\omega$ effects, we obtain

$\Gamma_{0}(t)=-\frac{\mathrm{i}}{2} \int \frac{d \Omega}{2 \pi} S(\Omega)\left[\frac{\sin ^{2} \theta(t)}{\Omega-B+\mathrm{i} 0^{+}}+\frac{2 \cos ^{2} \theta(t)}{\Omega+\mathrm{i} 0^{+}}\right]$

where the integral is along the real axis and the function $S(\Omega)$ is the Fourier transform of $S(\tau)$, it is real and even. The real part of $\Gamma_{0}$ is the dephasing rate, which is given by the residue of the poles at $\Omega=0, B$. The imaginary part of $\Gamma_{0}$ is the Lamb shift, coming from the principal value of the integral (only the $\sin ^{2} \theta$ term contributes).
Now we take into account the order- $\omega$ term in $\mathbf{B}^{\prime}(t)$ and the time-dependence of $\mathbf{B}^{\prime}(t)$; variations of the angle between $\mathbf{B}$ and $\mathbf{X}$ and of $B=|\mathbf{B}|$ [i.e. $\theta\left(t_{1}\right) \neq \theta(t)$ and $B(\tau) \neq B(t)$ in (9)]. To lowest order in $\omega$ the modification of the rate (10) are:

$$
\begin{aligned}
\delta_{\varphi} \Gamma(t) & =\mathrm{i} \dot{\varphi} \sin ^{2} \theta(t) \cos \theta(t) \times F(B), \\
\delta_{\theta} \Gamma(t) & =\mathrm{i} \dot{\theta} \sin \theta(t) \cos \theta(t) \times G(B), \\
\delta_{B} \Gamma(t) & =\mathrm{i} \dot{B} \sin ^{2} \theta(t) \times \frac{1}{2} G^{\prime}(B),
\end{aligned}
$$

the dot indicates $(\mathrm{d} / \mathrm{d} t)$, the prime indicates $(\mathrm{d} / \mathrm{d} B)$, and

$$
\begin{aligned}
& F(B) \equiv \mathrm{i} \frac{S(0)}{B}-\frac{1}{2} \int \frac{d \Omega}{2 \pi} \frac{S(\Omega)(3 B-2 \Omega)}{B\left(\Omega-B+\mathrm{i} 0^{+}\right)^{2}} \\
& G(B) \equiv \frac{\mathrm{i}}{2} \int \frac{d \Omega}{2 \pi}\left[\frac{S(\Omega)}{\left(\Omega-B+i 0^{+}\right)^{2}}-\frac{2 S(\Omega)}{\left(\Omega+\mathrm{i} 0^{+}\right)^{2}}\right] .
\end{aligned}
$$

From Eq. (7) one sees that these $\mathcal{O}[\omega]$-terms in $\Gamma(t)$ generate $\mathcal{O}\left[\omega^{0}\right]$-terms in the total phase acquired by $\rho_{12}: \delta \Phi_{\mathrm{BP}}=\int\left[F(B) \sin ^{2} \theta \cos \theta d \varphi+G(B) \sin \theta \cos \theta d \theta\right.$ $\left.+\frac{1}{2} G^{\prime}(B) \sin ^{2} \theta d B\right]$. This is geometric, and corresponds to $\delta \Phi_{\mathrm{BP}}=\int \mathrm{d} \mathbf{B} \delta \mathbf{a}(\mathbf{B})$ (cf. eq. (2)) with the complex 'vector potential'

$$
\begin{gathered}
\delta a_{\varphi}=B^{-1} F(B) \sin \theta \cos \theta, \\
\delta a_{\theta}=B^{-1} G(B) \sin \theta \cos \theta, \quad \delta a_{B}=\frac{1}{2} G^{\prime}(B) \sin ^{2} \theta,
\end{gathered}
$$

For open-paths of $\mathbf{B}(t)$ this is our main result, the imaginary part of this field (which gives geometric dephasing) is gauge-independent, while the real part is not. For closed paths of $\mathbf{B}(t)$ we use Stokes' theorem to write $\delta \Phi_{\mathrm{BP}}=\int d \mathbf{S} \delta \mathbf{b}(\mathbf{B})$ (cf. eq. (3)), finding that $\delta \mathbf{b}(\mathbf{B}) \equiv$ $\nabla_{\mathbf{B}} \times \delta \mathbf{a}$ has two non-zero components;

$$
\begin{aligned}
\delta b_{B} & =B^{-2} F(B)\left(3 \cos ^{2} \theta-1\right), \\
\delta b_{\theta} & =-B^{-1} F^{\prime}(B) \sin \theta \cos \theta .
\end{aligned}
$$

Thus it is independent of $\delta a_{\theta}$ and $\delta a_{B}$, their only role (for closed paths of $\mathbf{B}(t)$ ) is to form a 'pure gauge'; this is related to a symmetry discussed below. The angular dependence of $\delta \mathbf{b}$ resembles that of a quadrupole. For slow environment modes $(\Omega \ll B), \operatorname{Re}[F(B)] \propto B^{-2}$ and hence $\delta \mathbf{b}(\mathbf{B})$ is a quadrupole field. For other environment modes, $\delta \mathbf{b}(\mathbf{B})$ has non-zero curl and zero divergence. Thus it is not a sum multipoles, it is the field generated by a pseudo-current, $\mathbf{j}(\mathbf{B}) \equiv \nabla_{\mathbf{B}} \times \mathbf{b}(\mathbf{B})$, with one non-zero component

$$
j_{\varphi}(B)=B^{-3}\left(6 F(B)-B^{2} F^{\prime \prime}(B)\right) \sin \theta \cos \theta .
$$

Since we can ignore $\delta a_{\theta}$ and $\delta a_{B}$ for closed paths, eq. (13) leads to the following pretty result: the total (complex) $\mathrm{BP}$ for a closed path is

$$
\Phi_{\mathrm{BP}}=\oint \mathrm{d} \varphi \frac{\mathrm{d}}{\mathrm{d} B_{z}}\left(B+\delta B_{\mathrm{Lamb}}+\mathrm{i} T_{2}^{-1}\right) .
$$

This result can be understood as follows. For a timeindependent $\mathbf{B}$ the acquired phase is $\int d t \mathcal{E}$, where $\mathcal{E}$ is 
the term in parentheses in Eq. (17). When $\mathbf{B}$ is timedependent, $\mathbf{B} \rightarrow \mathbf{B}+\boldsymbol{\omega}$ in the RF changing the phase by $\int d t \omega \nabla_{\mathbf{B}} \mathcal{E}$; with Eq. (1) this gives Eq. (17).

Symmetry considerations. We now show that the vanishing of $b_{\varphi}$ follows from the symmetries of the problem. Firstly, under time-reversal $\mathbf{B}(t), \mathbf{X}(t) \rightarrow$ $-\mathbf{B}(-t),-\mathbf{X}(-t)$ the BP between the excited and ground states is invariant, while in eq. (3) $d \mathbf{S} \rightarrow-d \mathbf{S}$. This implies that $\mathbf{b}(-\mathbf{B},-X)=-\mathbf{b}(\mathbf{B}, X)$. Secondly, if we instead rotate all spins and fields by angle $\pi$ about the axis $\hat{\varphi}$ perpendicular to both $\mathbf{B}$ and $X($ e.g. $\mathbf{B} \rightarrow-\mathbf{B})$, the field $\mathbf{b}$ would also rotate. Thus, $\mathbf{b}(-\mathbf{B},-X)=$ $R_{\varphi}^{\pi} \mathbf{b}(\mathbf{B}, X)$. To satisfy both equalities, $b_{\varphi} \equiv 0$.

For isotropic coupling, the Hamiltonian's rotational symmetry guarantees that $b_{\varphi}=b_{\theta}=0$. The monopole (cf. eq. (3) ) is quantized so $\delta b_{B}=0$; hence $\delta \Phi_{\mathrm{BP}}=0$ to all orders in the coupling to the environment.

Contribution of the slow modes $(\Omega \ll B)$. Here $F(B) \propto B^{-2}$, so $\delta \mathbf{b}$ is a quadrupole field. This effect of (for instance, classical) slow modes can be understood by noting that $(\mathbf{B}(t)+\mathbf{X}(t))$ varies adiabatically, hence

$$
\Phi_{\mathrm{BP}}=\oint d(\mathbf{B}+\mathbf{X}) \mathbf{a}_{\mathrm{m}}(\mathbf{B}+\mathbf{X})=\oint d \mathbf{B} \mathbf{a}_{\mathrm{m}}(\mathbf{B}+\mathbf{X}),
$$

where $\mathbf{a}_{\mathrm{m}}$ is the field of a charge-one monopole at the origin. We disregard boundary corrections due to $\mathbf{X}\left(t_{\mathrm{P}}\right) \neq \mathbf{X}(0)$ and drop the term $\oint d \mathbf{X} \mathbf{a}_{\mathrm{m}}(\mathbf{B}+\mathbf{X})[27]$. The remaining term is the field of a monopole at the point $-\mathbf{X}$. In the multipole expansion, $\mathbf{a}_{\mathrm{m}}(\mathbf{B}+\mathbf{X})=$ $\mathbf{a}_{\mathrm{m}}(\mathbf{B})+X_{\alpha} \nabla_{\alpha} \mathbf{a}_{\mathrm{m}}(\mathbf{B})+\frac{1}{2} X_{\alpha} X_{\beta} \nabla_{\alpha} \nabla_{\beta} \mathbf{a}_{\mathrm{m}}(\mathbf{B})+\cdots$. The first term produces the unperturbed $\mathrm{BP}$, the second (dipole) term vanishes after averaging over the fluctuations 11]. Thus the quadrupole term gives the leading environment-induced modification of the BP. For noise along the $z$-axis this term reads $D_{\alpha \beta} \nabla_{\alpha} \nabla_{\beta} \mathbf{a}_{\mathrm{m}} / 6$, with the quadrupole moment $D_{\alpha \beta}=\left\langle X^{2}\right\rangle \operatorname{diag}(-1,-1,2)$.

Concerning experiments. Our results imply that the traversal of the same loop $\mathbf{B}(t)$ in opposite directions would yield, apart from different BP's, different dephasing. We further note that a noise spectrum suppressed at $\Omega=0, B$ will increase $T_{2}$ while having little effect on $\delta \Phi_{\mathrm{BP}}$, thus aiding the observation of $\delta \Phi_{\mathrm{BP}}$.

This work is part of CFN (DFG), supported in part by the Minerva (DFG) Foundation, the EC-RTN Spintronics, and ISF of IAS. RW, YM and YG were supported by the EPSRC, the Dynasty Foundation and the AvH Foundation (Max-Planck award) respectively. The Transnational Access prog. supported the WIS visit of YM,AS.

[1] M. V. Berry, Proc. R. Soc. Lond. 392, 45 (1984).

[2] R.S. Whitney and Y. Gefen, Phys. Rev. Lett. 90, 190402 (2003).

[3] Y. Nakamura, Yu. A. Pashkin, and J. S. Tsai, Nature 398, 786 (1999).
[4] D. Vion, A. Aassime, A. Cottet, P. Joyez, H. Pothier, C. Urbina, D. Esteve, and M. H. Devoret, Science 296, 886 (2002).

[5] I. Chiorescu, Y. Nakamura, C. J. P. M. Harmans, and J. E. Mooij, Science 299, 1869 (2002).

[6] G. Falci, R. Fazio, G. H. Palma, J. Siewert, and V. Vedral, Nature 407, 355 (2000).

[7] All energies and magnetic fields are in units of inverse time $\left(g \mu_{\mathrm{B}}=\hbar=1\right)$.

[8] J. C. Garrison and E. M. Wright, Phys. Lett. A 128, 177 (1988).

[9] see e.g. D. Ellinas, S. M. Barnett, and M. A. Dupertuis, Phys. Rev. A 39, 3228 (1989), D. Gamliel, J. H. Freed, Phys. Rev. A 39, 3238 (1989); A. Carollo, I. FuentesGuridi, M.F. Santos, and V. Vedral, Phys. Rev. Lett. 90, 160402 (2003).

[10] F. Gaitan, Phys. Rev. A 58, 1665 (1998), J.E. Avron, A. Elgart, Phys. Rev. A 58, 4300 (1998).

[11] G. De Chiara and G. M. Palma, Phys. Rev. Lett. 91, 090404 (2003) consider only the dipole slow-noise term and thus do not find $\delta \Phi_{\mathrm{BP}}$, cf. below our eq. (18).

[12] Here we ignore the boundary terms, $\Phi_{\text {shift }}$; cf. [2].

[13] If $\mathbf{B}(t)$ traverses a loop $n$ times in a time $t_{\mathrm{P}} \sim T_{2}$, the leading non-adiabatic correction $\sim n^{2}\left\langle\hat{X}^{2}\right\rangle$, while $\delta \Phi_{\mathrm{BP}} \sim n\left\langle\hat{X}^{2}\right\rangle$. We can separate the two by varying $t_{\mathrm{P}}$ (keeping $t_{\mathrm{P}} \sim T_{2}$ ) or $n$. Alternatively the environment's spectrum may make non-adiabatic effects $\ll \operatorname{Re}\left[\delta \Phi_{\mathrm{BP}}\right]$, because $\delta \Phi_{\mathrm{BP}}$ comes from the whole spectrum while $T_{2}^{-1}$ is dominated by frequencies $0, B$ (see below eq. (10).

[14] F. Wilczek and A. Zee, Phys. Rev. Lett. 52, 2111 (1984).

[15] M.V. Berry, Proc. R. Soc. Lond. A, 414, 31 (1987).

[16] To avoid confusion we define $\hat{\boldsymbol{\sigma}} \equiv\left(\hat{\sigma}_{x}, \hat{\sigma}_{y}, \hat{\sigma}_{z}\right)$ as the same Pauli matrices, e. g. $\hat{\sigma}_{z}=\operatorname{diag}(1,-1)$, in all frames.

[17] Typically $(\tilde{\mathbf{B}}+\tilde{\boldsymbol{\omega}})$ in the RF is non-stationary and is not quite parallel to the $\tilde{z}$-axis. This only gives non-adiabatic corrections, $\mathcal{O}\left(\omega^{2} / B, \dot{\omega} / B\right)$; cf. [15].

[18] For multi-directional coupling $\delta \Phi_{\mathrm{BP}}$ is the sum of the $\delta \Phi_{\mathrm{BPS}}$ calculated separately for each coupling axis to $\mathcal{O}\left[\left\langle\hat{X}^{2}\right\rangle\right]$. We find $\delta \Phi_{\mathrm{BP}}=0$ for isotropic coupling.

[19] see e.g. U. Weiss, Quantum dissipative systems (World Scientific, Singapore, 1999). C. Cohen-Tannoudji, J. Dupont-Roc and G. Grynberg, Atom-photon interactions (Wiley, New York, 1992).

[20] F. Bloch, Phys. Rev. 105, 1206 (1957).

[21] A. G. Redfield, IBM J. Res. Dev. 1, 19 (1957).

[22] H. Schoeller and G. Schön, Phys. Rev. B 50, 18436 (1994).

[23] For an introduction see Yu. Makhlin, G. Schön, and A. Shnirman in New Directions in Mesoscopic Physics Edited by V.F. Gantmakher, R. Fazio, and Y. Imry (Kluwer Academic, Dordrecht, 2003) p. 197.

[24] L. D. Landau and E. M. Lifshitz, Quantum Mechanics (Pergamon, Oxford, 1974), chap. VI.

[25] The above integral is dominated by $t_{1}$ close to $t$ thus we can take the lower bound of integration to $-\infty$ with little effect (except at small $t$ where it causes a small "preparation" correction).

[26] R. S. Whitney, Yu. Makhlin, A. Shnirman, and Y. Gefen, in cond-mat/0401376 (2004).

[27] Since for $\mathbf{X} \| \boldsymbol{e}_{z}$ in the chosen gauge $d \mathbf{X} \perp \mathbf{a}_{\mathrm{m}}$; this also holds for general $\mathbf{X}$ as long as $\mathbf{X}(t)$ and $\mathbf{X}(-t)$ have equal weights since their contributions cancel. 